\title{
Diagnostic Value of the Acid-Labile Subunit in Acromegaly: Evaluation in Comparison with Insulin-Like Growth Factor (IGF) I, and IGF-Binding Protein-1, -2, and -3*
}

\author{
M. AROSIO, S. GARRONE, P. BRUZZI, G. FAGLIA, F. MINUTO, ANd A. BARRECA \\ Institute of Endocrine Sciences, University of Milan, Ospedale Maggiore Istituto Ricovero e Cura a \\ Carattere Scientifico (M.A., G.F.), I-20122 Milan; and Department of Endocrinology and Metabolism, \\ University of Genova (S.G., A.C., F.M., A.B.), and Unit of Clinical Epidemiology and Trials, National \\ Institute for Cancer Research (P.B.), I-16132 Genova, Italy
}

\begin{abstract}
In normal subjects the main form of circulating insulin-like growth factor (IGF) is the $150-\mathrm{kDa}$ complex. This complex is formed by the IGF peptide, the acid-stable IGF-binding protein-3 (IGFBP-3), and the acid-labile subunit (ALS). Experimental and clinical data have demonstrated that ALS is primarily under the control of GH and plays a critical role in maintaining constant levels of circulating IGF-I. In this study we evaluated ALS, IGF-I, and IGFBP-1, -2, and -3 in 45 acromegalic patients in basal conditions and, in 37 of these, twice after surgical therapy compared with 100 age- and sex-matched control subjects to estimate their value as parameter of GH secretory state.

The results demonstrated that in acromegaly before treatment all parameters (ALS, $523 \pm 26$; IGF-I, $129 \pm 6$; IGFBP-1, $0.7 \pm 0.1$; IGFBP-3, $234 \pm 21$; nmol/L; mean \pm SEM) but IGFBP-2 were significantly different $(P<0.0001)$ from those in healthy subjects (ALS, $281 \pm 4$; IGF-I, $22 \pm 1$; IGFBP-1, $1.6 \pm 0.1$; IGFBP-3, $91 \pm 3$ ). IGF-I was more sensitive $(100 \%)$ than ALS $(89 \%)$, and both were more predictive of disease status than IGFBP-3, in that $27 \%$ of the patients had IGFBP-3 levels within the normal range. Considering the ALS/ IGFBP-3 molar ratio, almost 55\% of ALS circulated in a free form in active acromegaly. Before treatment, the IGF-I/IGFBPs $(-1+-2+$ -3) molar ratio, which can be regarded as free, biologically active, IGF-I, was greatly increased $(0.77 \pm 0.06 ; P<0.0001)$ compared with that in control subjects $(0.23 \pm 0.01)$.
\end{abstract}

After surgery, all 10 patients with controlled disease showed normalization of ALS (100\% sensitivity), whereas 9 of them had normal IGFBP-3; reevaluation after varying lengths of time showed all these parameters within the normal range. In the 27 patients with active disease, IGF-I and ALS were more predictive of disease status (91\% and $83 \%$ negative predictive values, respectively) than IGFBP-3 $(53 \%)$.

The basal ALS concentration correlated only with IGFBP-3 $(\mathrm{r}=$ $0.70 ; P<0.001$ ). In postsurgery samples (first control) a statistically significant $(P<0.001)$ correlation was found between mean $\mathrm{GH}$ values as well as minimum GH after oral glucose tolerance test and ALS $(\mathrm{r}=0.72$ and 0.83 , respectively), IGF-I ( $\mathrm{r}=0.69$ and 0.77$)$, IGFBP-3 $(\mathrm{r}=0.50$ and 0.72$)$, and IGFBP-2 $(\mathrm{r}=-0.36$ and -0.63$)$. Similarly, IGF-I, IGFBP-3, and ALS were positively correlated among themselves and negatively correlated with IGFBP-2 $(P<0.001)$.

In conclusion, in the diagnosis of acromegaly, the measurement of total IGF-I appears to be the most sensitive parameter among the subunits of the 150K complex, and IGFBP-3 the least sensitive. For ALS, this subunit is quite sensitive and appears to be a useful parameter in reassessment after surgical treatment. (J Clin Endocrinol Metab 86: 1091-1098, 2001)

which does not by itself bind IGF-I, but is necessary to reconstitute the whole complex from purified components (3-6). The ALS has been shown to be a glycosylated protein of $84-86 \mathrm{kDa}$ and appears to have the role of increasing the molecular weight of the IGF-IGFBP-3 complex, which results in a prolongation of the IGF half-life to about $15 \mathrm{~h}(4,5)$. This latter aspect is of great importance, as the IGF peptide does not readily cross the capillary-endothelial barrier in its 150$\mathrm{kDa}$ form, and the ALS is therefore essential for ensuring constant plasma IGF levels (7). However, IGF-I measurement presents methodological problems, mostly due to its association with the IGFBPs in circulation. Current methods of minimizing IGFBP interference in routine assays are complex, troublesome, and often inefficient (8-10). The IGFBP-3 assay is simpler. However, IGFBP-3 concentrations are variable in acromegalic disease and do not seem always to reflect the activity of the disease $(11,12)$.

Despite the recognized GH dependence of the ALS and the stability of its circulating concentration, few data are cur- 
rently available with regard to its reliability as a parameter of GH secretory status, and these are often conflicting (1315). Experimental and clinical data demonstrate that the ALS, like IGF-I, is primarily under the control of GH (16-18). Except in critically ill patients (19-21), the ALS is relatively independent of mechanisms known to influence IGF-I and IGFBP-3 secretion (22), thus suggesting that it might be a more specific parameter of GH secretory status. Moreover, as its measurement is unaffected by IGFBPs, which may interfere in many conventional IGF-I assays (23), it could offer several advantages over IGF-I in monitoring the activity of acromegalic disease. However, ALS measurement is relatively recent, and data on ALS concentrations in acromegaly are very scarce (23-25).

The aim of the present study is to compare ALS levels in a group of healthy subjects with those of a group of patients with active acromegaly. The effects of both successful and unsuccessful pituitary surgery on ALS levels, as judged by GH suppression during an oral glucose tolerance test (OGTT), are also reported. The reliability of the ALS in acromegaly will be considered in comparison with other components of the GH-IGF-I axis: GH, IGF-I, IGFBP-1, IGFBP-2, and IGFBP-3.

\section{Materials and Methods \\ Patient population and protocol}

Forty-five acromegalic patients ( 23 women and 22 men; age, 21-64 yr) who had undergone adenomectomy were included in this study. Acromegaly was diagnosed on the basis of clinical features, high serum IGF-I concentrations compared with the age-adjusted normal range, and elevated GH levels that were not suppressible to less than $1 \mu \mathrm{g} / \mathrm{L}$ during an OGTT (26). The duration of the acromegalic disease ranged from 1-20 yr (median, 6 yr). All patients were off medical therapy for acromegaly, two were receiving replacement therapy with $\mathrm{T}_{4}$, and none was taking glucocorticoids. Most patients had been newly diagnosed. Four patients who had received octreotide therapy (300 $\mu \mathrm{g} /$ day, sc) were assessed at least 1 month after therapy discontinuation. GH concentrations (mean of at least four fasting samples) were $23.6 \pm 3.2 \mu \mathrm{g} / \mathrm{L}$ ( $\pm \mathrm{SEM})$, ranging from $2.6-78.5 \mu \mathrm{g} / \mathrm{L}$. In 37 patients PRL levels were within the normal range $(<20 \mu \mathrm{g} / \mathrm{L}$ in women and $<15 \mu \mathrm{g} / \mathrm{L}$ in men), whereas 8 patients had elevated PRL levels ranging from $23-108 \mu \mathrm{g} / \mathrm{L}$. Gonadal function was normal in 15 men and 16 women. Six women were in the menopause and were not receiving hormone replacement therapy; 1 was amenorrheic. Seven men had reduced testosterone levels ranging from 2.4-9.1 nmol/L (normal range, 13-42). Two patients had diabetes mellitus: 1 was treated with insulin, and 1 with oral antidiabetic agents. In all patients the presence of a GH-secreting tumor was confirmed by immunohistochemical studies of surgically removed tissue.

Thirty-seven patients were reevaluated 1-6 months after surgery (first control). None of them had undergone medical therapy. Ten patients were considered controlled on the basis of GH suppression to less than $1 \mu \mathrm{g} / \mathrm{L}$ after OGTT and normal IGF-I concentrations compared with the age-adjusted normal range. In these 10 patients, the abnormal GH responses present before surgery (to TRH in 7, to sulpiride injected during dopamine infusion in 2, and to GnRH in 1) had also disappeared. One patient presenting with IGF-I level above the 97th percentile of the control group and nadir GH after OGTT below $1 \mu \mathrm{g} / \mathrm{L}$ was considered to have persistent disease. Two other patients, in whom GH decreased to $1 \mu \mathrm{g} / \mathrm{L}$ and not less than 1 after OGTT while abnormal GH responses were maintained, were not considered cured (27). Gonadal function normalized in 3 men and worsened in 2; all other anterior pituitary functions were unchanged. Antidiabetic therapies were unmodified.

In 4 of 10 patients with controlled disease and in 21 of 27 with active disease, further evaluation (second control) was performed 12-36 months after surgery. The uncontrolled group of patients was assessed while off medical therapy. Seven of these patients had undergone radiotherapy in the meantime.

One hundred age- and sex-matched healthy blood donors volunteered as controls (Table 1)

In all patients and normal controls, venous blood samples were taken in the morning (after overnight fasting) for the determination of IGF-I, ALS, IGFBP-1, IGFBP-2, and IGFBP-3. Serum was kept at $-20 \mathrm{C}$ until assayed. All pre- and postoperative samples from the same subject were assessed in the same assay.

\section{Analytical methods}

Serum GH levels were determined by an immunofluorimetric assay (AutoDelfia hGH, Wallac, Inc., Turku, Finland). The sensitivity of the assay was $0.01 \mu \mathrm{g} / \mathrm{L}$; the intra- and interassay coefficients of variation were $2.6 \%$ and $3.9 \%$, respectively.

IGF-I was measured by RIA using immunochemicals and tracer provided by Medgenix (Fleurus, Belgium). The sensitivity of the assay was $1.2 \mathrm{nmol} / \mathrm{L}$; the intra- and interassay coefficients of variation were $6 \%$ and $7.5 \%$, respectively. To avoid interference from binding proteins, single plasma ethylenediamine tetraacetate samples were treated with acid-ethanol according to the method of Daughaday et al. (28).

Serum total ALS was measured by means of specific two-site sandwich enzyme-linked immunosorbent assay, using anti-ALS antibodies raised against synthetic amino-terminal and carboxyl-terminal ALS peptides, and reagents and tracer provided by Diagnostics Systems Laboratories, Inc. (Webster, TX). All samples were pretreated to dissociate the complexed ALS and enhance ALS immunoreactivity. The sensitivity of the assay was $4.7 \mathrm{nmol} / \mathrm{L}$; the intra- and interassay coefficients of variation were $5.5 \%$ and $7.2 \%$, respectively. Recovery of human serumderived glycosylated ALS [purified as described previously (6)] was 75\% for the lower concentration added $(1 \mu \mathrm{g})$ and $95 \%$ for the higher concentration added $(60 \mu \mathrm{g})$.

IGFBP-1 was measured by immunoradiometric assay, using reagents and tracer provided by Diagnostics Systems Laboratories, Inc. The sensitivity of the assay was $0.05 \mathrm{nmol} / \mathrm{L}$; the intra- and interassay coefficients of variation were $2.5 \%$ and $4.6 \%$, respectively.

IGFBP-2 levels were determined by double antibody RIA using a nonequilibrium technique, as described by Clemmons et al. (29). Specific IGFBP-2 antiserum was purchased from Upstate Biotechnology, Inc. (Lake Placid, NY), and the standard was a pure IGFBP-2 preparation obtained by DNA recombinant technology (ImmunoKontact, Frankfurt, Germany). The sensitivity of the assay was $0.01 \mathrm{nmol} / \mathrm{L}$; the intra- and interassay coefficients of variation were $6 \%$ and $9.5 \%$, respectively.

IGFBP-3 was measured by immunoassay, using reagents and tracer

TABLE 1. Comparison between hormone levels in healthy subjects and in untreated acromegalic patients

\begin{tabular}{lcc}
\hline \multicolumn{1}{c}{ Parameters } & Healthy subjects & Acromegalic patients \\
\hline No. of cases & 100 & 45 \\
Sex (F/M) & $0 / 50$ & $23 / 22$ \\
Age (yr) & $43 \pm 12(40-45)$ & $39 \pm 11(36-42)$ \\
ALS (nmol/L) & $281 \pm 43(273-290)$ & $523 \pm 171(473-573)$ \\
IGF-I (nmol/L) & $22 \pm 4(21-23)$ & $129 \pm 39(118-140)$ \\
IGFBP-1 (nmol/L) & $1.6 \pm 1.2(1.4-1.9)$ & $0.71 \pm 0.6(0.5-0.9)$ \\
IGFBP-2 (nmol/L) & $8.6 \pm 5.4(7.6-9.7)$ & $7.5 \pm 5.8(5.7-9.1)$ \\
IGFBP-3 (nmol/L) & $91 \pm 29(86-97)$ & $234 \pm 141(193-275)$ \\
\hline
\end{tabular}

Data are shown as the mean \pm SD; $95 \%$ confidence intervals are given in parentheses. 
provided by Diagnostics Systems Laboratories, Inc. All samples were diluted appropriately so as to reach a point in the curve where there is parallelism among unglycosylated Escherichia coli-derived IGFBP-3, glycosylated Chinese hamster ovary-derived IGFBP-3, and serum $(\% \mathrm{~B} / \mathrm{B} 0$, $70-85 \%)$. The sensitivity of the assay was $0.04 \mathrm{nmol} / \mathrm{L}$; the intra- and interassay coefficients of variation were $3.25 \%$ and $5.6 \%$, respectively.

\section{Statistical analysis}

Statistical analysis was performed by nonparametric test on paired (Wilcoxon signed rank test) and unpaired (Mann-Whitney test) observations. $P<0.05$ was considered significant. Results are expressed as the mean \pm SEM. The correlations among all parameters studied were evaluated using Spearman rank order statistics and linear regression analysis models. For this purpose, log-transformed values of $\mathrm{GH}$ and IGFBP-3, which showed a log normal distribution, were used. The 97th percentile of the normal distribution calculated for each age group in the healthy subjects was chosen as the cut-off point for normal serum levels of all parameters of the IGF system. Because normal levels are largely arbitrary and vary according to laboratory, we investigated the diagnostic accuracy of each parameter at different cut-off points by determination of the receiver-operating characteristic (ROC). All statistical analyses were made using SPSS statistical software (SPSS, Inc., Chicago, IL).

\section{Results}

\section{Healthy subjects}

The mean concentrations of the different parameters are shown in Table 1 and Fig. 1. Analytical distribution of age and single values of IGF-I, IGFBP-3, and ALS are shown in Fig. 2.

As expected, a negative correlation between IGF-I and age and between IGFBP-3 and age $(\mathrm{r}=-0.61$ for IGF-I and $\mathrm{r}=$ -0.40 for IGFBP-3; $P<0.001$ for both) was observed. No statistically significant correlation was found between ALS levels and age in the 20-65 yr age range. Indeed, a statistically significant difference $(P<0.001)$ between 20 - to $35-y r-$ old subjects and 50- to 65-yr-old subjects was present for IGF-I, IGFBP-2, and IGFBP-3, but not for ALS. IGFBP-2 levels significantly increased with age in women, but not in men $(\mathrm{r}=0.51 ; P<0.01)$. No other gender differences were found. ALS concentrations were significantly correlated with IGF-I $(P<0.0001)$ and IGFBP-3 levels $(P<0.001)$. The ALS/ IGFBP-3 molar ratio was $3.4 \pm 0.1$. Overall, the 3 rd and 97 th percentiles were 13.3 and $30.1 \mathrm{nmol} / \mathrm{L}$ for IGF-I, 216 and 350 $\mathrm{nmol} / \mathrm{L}$ for ALS, 50 and $136 \mathrm{nmol} / \mathrm{L}$ for IGFBP-3, 0.2 and 3.9 $\mathrm{nmol} / \mathrm{L}$ for IGFBP-1, and 2.4 and $18.7 \mathrm{nmol} / \mathrm{L}$ for IGFBP-2.

\section{Acromegalic patients: before surgery}

In the group of acromegalic patients ALS levels were elevated before transsphenoidal surgery (Table 1 and Figs. 1 and 2). ALS levels were higher than the 97th percentile of healthy subjects in 40 patients (89\%). One of the 5 patients in
FIG. 1. Serum levels of IGF-I, ALS, IGFBP-3, IGFBP-1, and IGFBP-2 in 100 normal subjects, 45 acromegalic patients before surgery, and 37 acromegalic patients after surgery (11 with controlled and 26 with active disease). Data are shown as the mean \pm SEM. $\bullet$, $P<0.05$; $\bullet, P<0.001 ; 0 \bullet 0$ or $* * *$, $P<0.0001$.
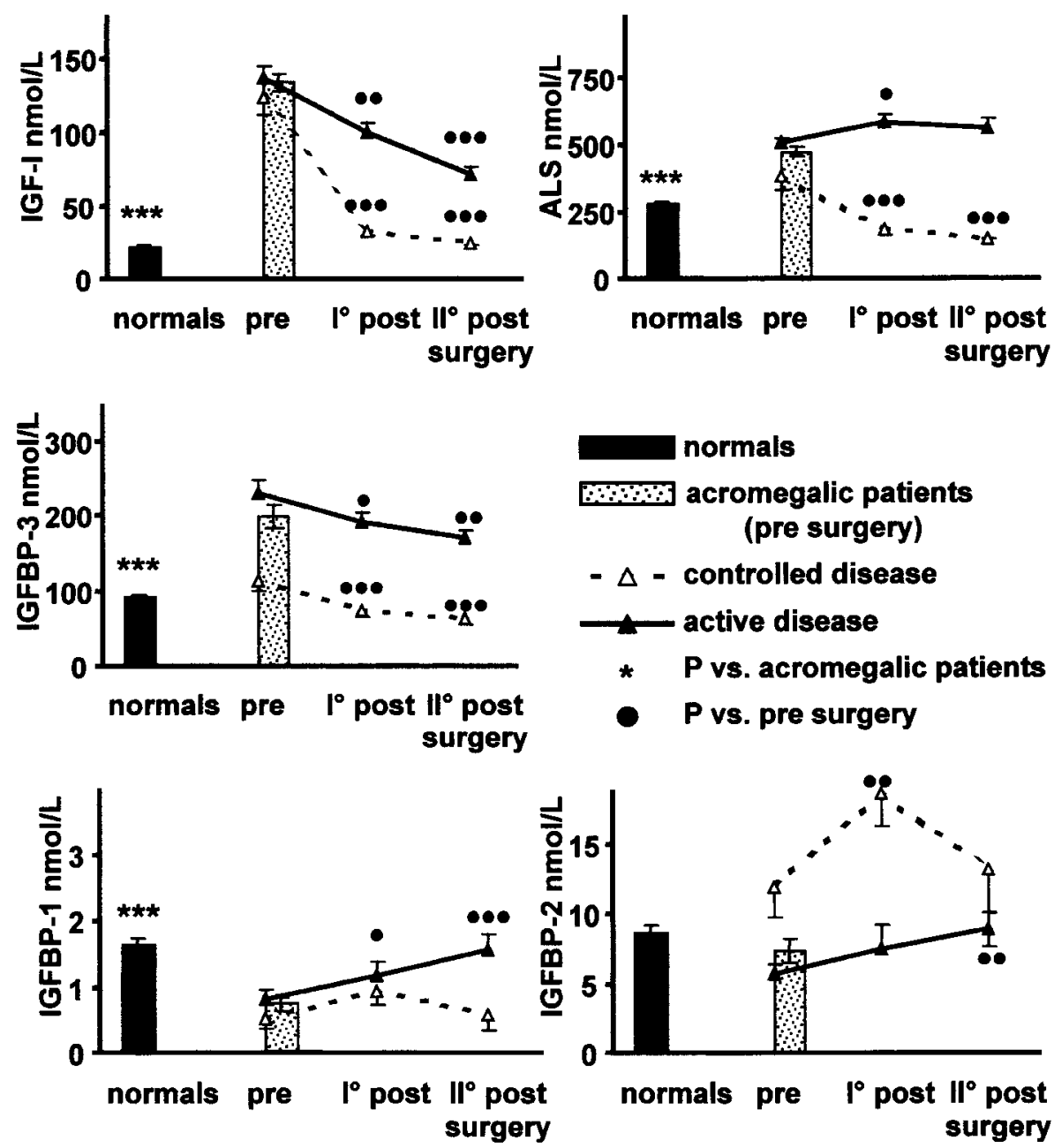


\section{BEFORE SURGERY \\ - acromegalic patients o normals}
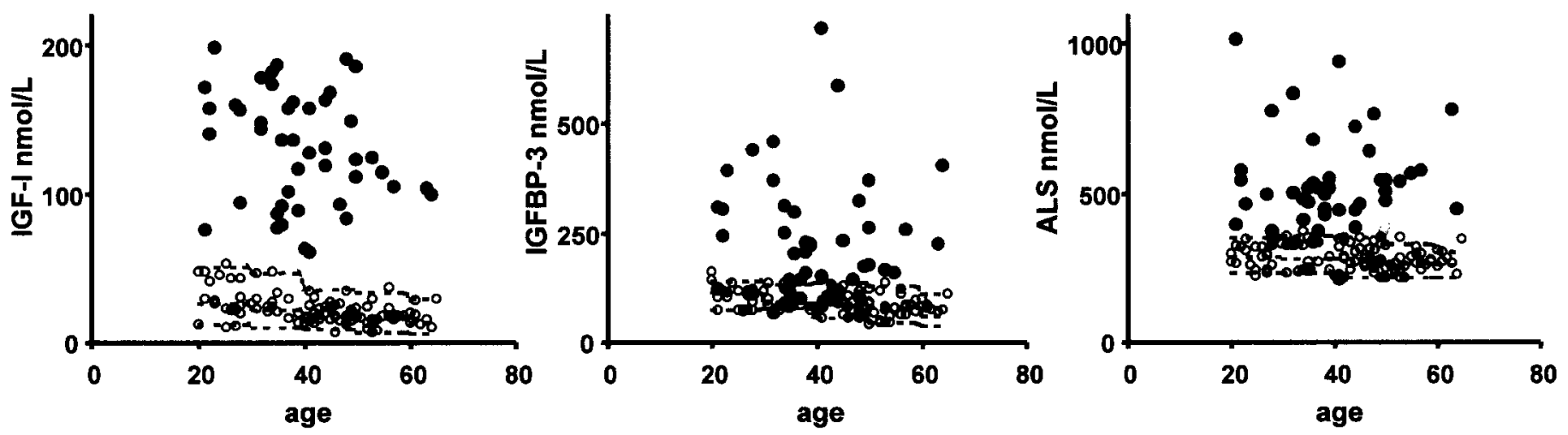

AFTER SURGERY

- active disease

o controlled disease
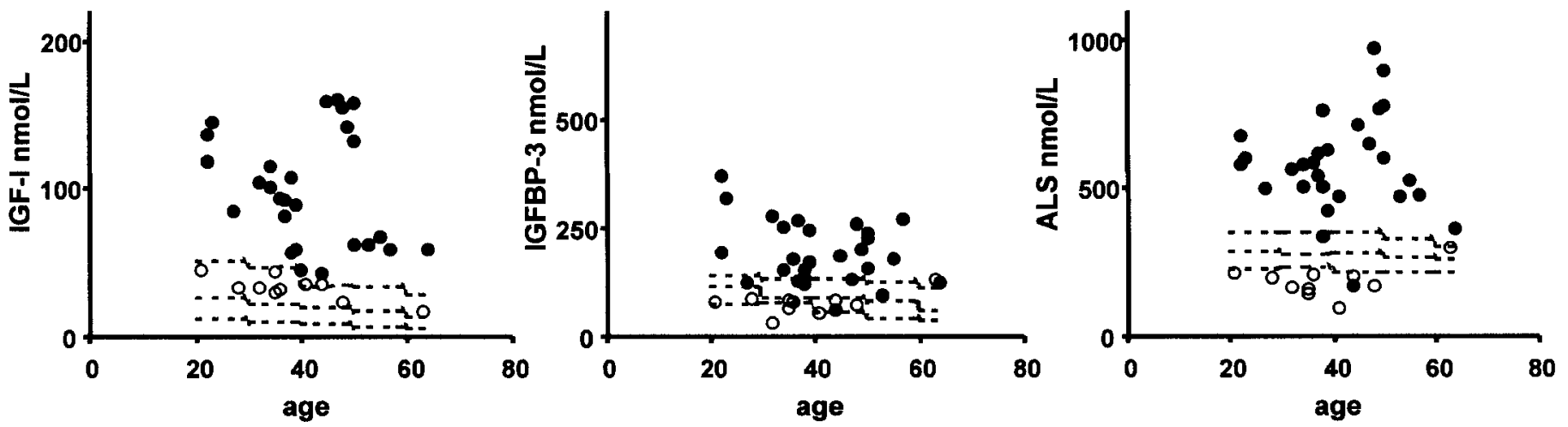

FIG. 2. Serum IGF-I, IGFBP-3, and ALS in normal subjects and in 45 acromegalic patients before and after surgery. Dashed lines represent the 3rd, 50th, and 97 th percentiles of the control group.

whom ALS levels were below the 97th percentile of healthy controls also showed the lowest concentration of GH (mean value, $2.6 \mu \mathrm{g} / \mathrm{L})$ and response after OGTT $(1.4 \mu \mathrm{g} / \mathrm{L})$. IGF-I concentration, although always above the normal range for age, tended to be lower in these patients than in patients with high ALS levels. All 5 also showed IGFBP-3 below the 97th percentile and IGFBP-2 levels in the higher range concentration. The basal ALS concentration correlated with IGFBP-3 $(\mathrm{r}=0.70 ; P<0.001)$. No correlation with age, GH, IGF-I, IGFBP-1, IGFBP-2, or tumor size was observed in untreated acromegalic patients. No differences according to gender, PRL levels, or gonadal status were observed.

IGF-I levels were elevated in all patients compared with those in normal controls (Table 1 and Figs. 1 and 2), ranging from 60-197 nmol/L. The IGF-I/IGFBPs $(-1+-2+-3)$ molar ratio was significantly $(P<0.001)$ higher $(0.77 \pm 0.1)$ than that in normal subjects $(0.23 \pm 0.01)$. In contrast with healthy subjects, the IGF-I concentration did not decrease with age. A significant correlation was found between IGF-I and GH mean concentrations and nadir value after OGTT ( $\mathrm{r}=0.44$ and $0.59 ; P<0.01)$.

IGFBP-3 levels ranged from 67-717 nmol/L (Table 1 and Figs. 1 and 2). About two thirds of the patients showed high IGFBP-3 concentrations, whereas 12 (27\%) had IGFBP-3 lev- els in the normal range. No correlations were found with the parameters evaluated, apart from ALS.

IGFBP-1 concentrations ranged from $0.08-2.46 \mathrm{nmol} / \mathrm{L}$ and were significantly lower than IGFBP-1 levels in normal controls (Table 1 and Fig. 1).

IGFBP-2 levels ranged from $1.44-26.37 \mathrm{nmol} / \mathrm{L}$ and were not statistically different from those in controls (Fig. 1). However, with regard to the IGFBP-2/IGF-I molar ratio, 95\% of patients showed a value below the third percentile of that in healthy subjects.

None of the parameters considered, apart from GH concentration $(P<0.01)$, correlated with the size of the adenoma.

Information on the discriminatory ability of each parameter evaluated by ROC curve analysis (Fig. 3) was in close agreement with the estimates obtained using the cut-off points from normal controls (97th percentile).

No ROC curve for IGF-I could be obtained, as discrimination between acromegalic patients and normal controls was total, with no overlapping of the values in the two groups. The areas under the curve for ALS, IGFBP-3, IGFBP-1, and IGFBP-2 were $0.937,0.892,0.798$, and 0.584 , respectively, indicating that the parameter with the best discrimination, after IGF-I, was ALS, followed by IGFBP-3. 
ALS
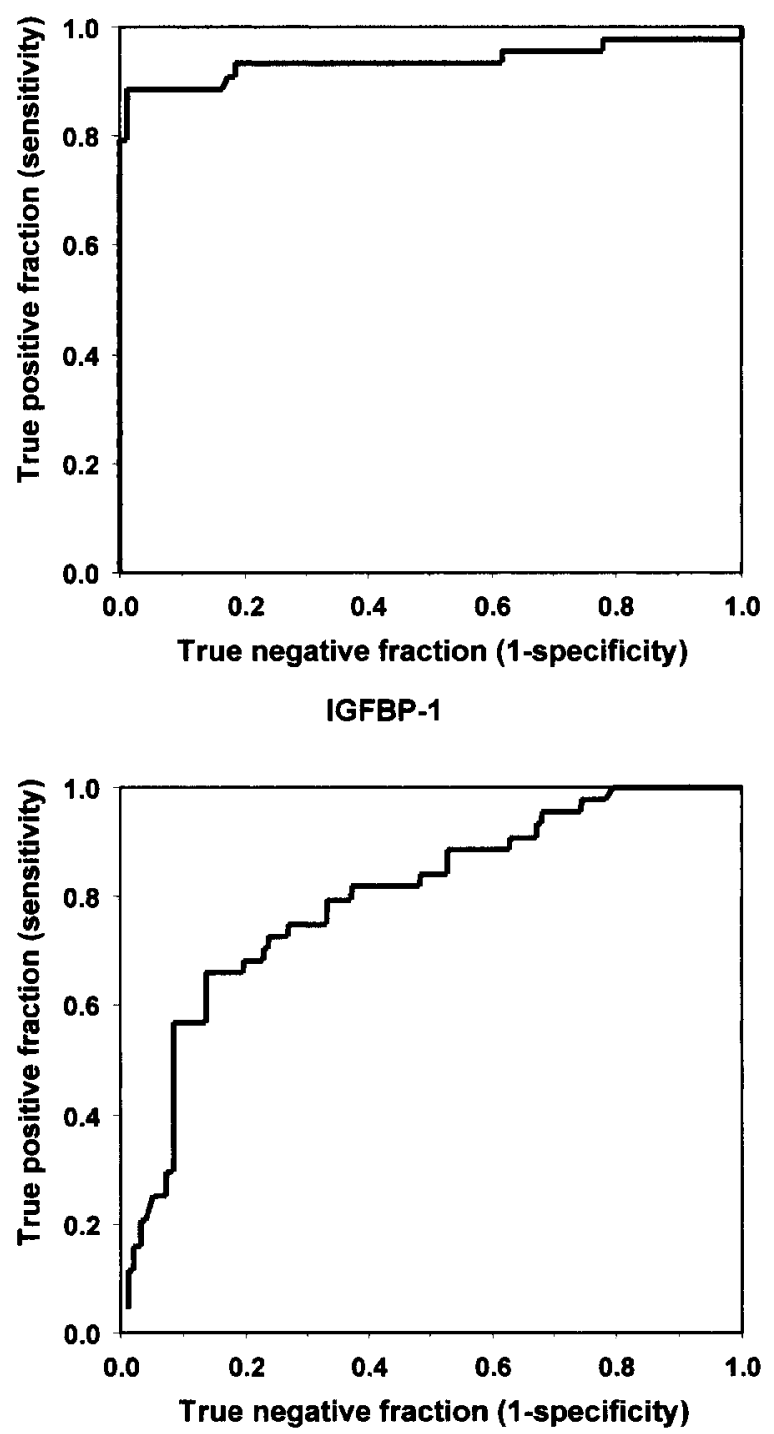

IGFBP-3
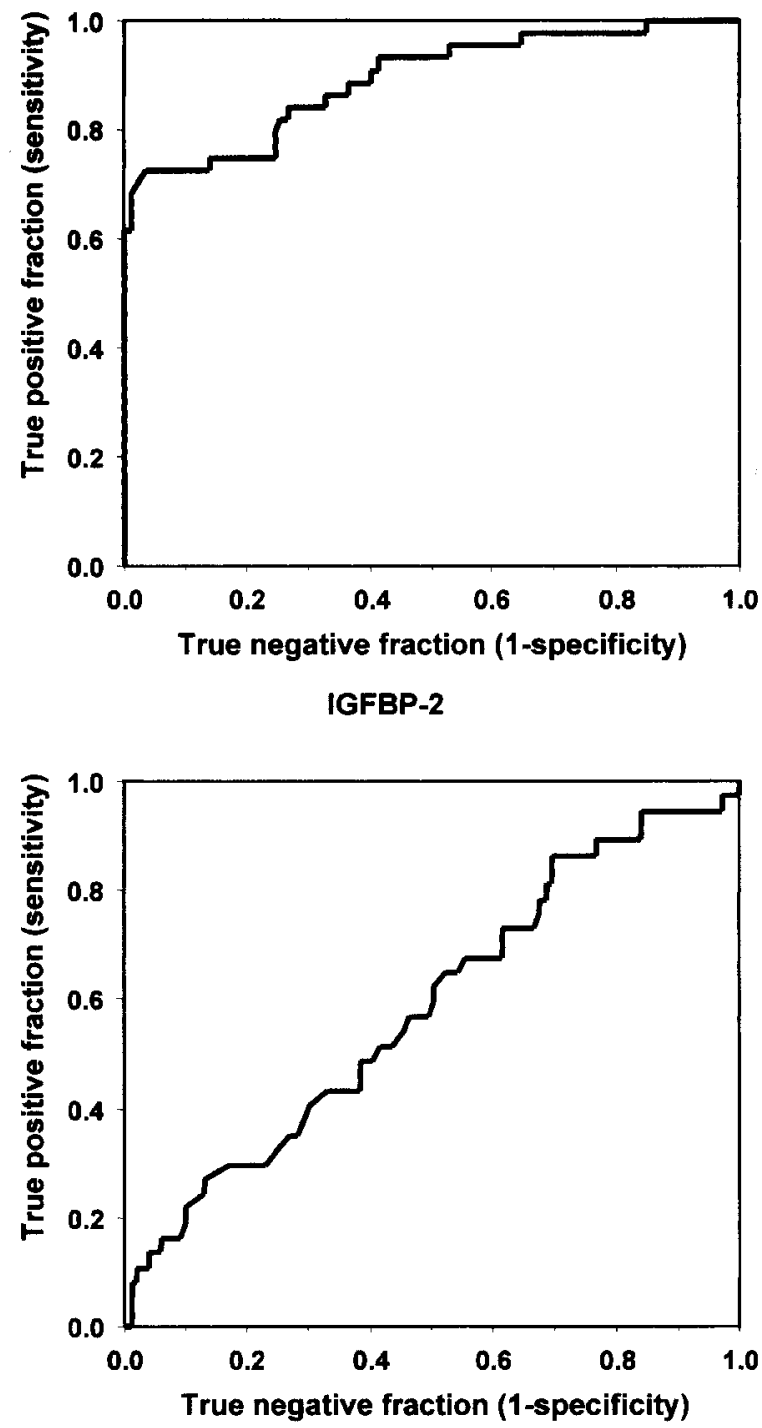

FIG. 3. ROC assessed the comparative diagnostic performance of ALS, IGFBP-3, IGFBP-1, and IGFBP-2. The ROC curve for IGF-I cannot be estimated because there was no overlap between values in cases and in controls (sensitivity, 100\%; specificity, 100\%).

\section{Acromegalic patients: reevaluation after surgery}

Thirty-seven patients were reevaluated after transsphenoidal surgery. Mean levels of the different parameters before and after surgery are shown in Fig. 1. All parameters showed the expected modifications after removal or reduction of the adenoma; GH, IGF-I, and IGFBP-3 decreased, whereas IGFBP-1 and IGFBP-2 increased. The sensitivity, specificity, and positive and negative predictive values of ALS, IGF-I, and IGFBP-3 in identifying patients with controlled and active disease are shown in Table 2.

Surgery was considered successful in 10 patients, in whom an oral glucose tolerance test suppressed GH concentrations below $1 \mu \mathrm{g} / \mathrm{L}$, and IGF-I was normalized. Before surgery, this group of patients had significantly lower levels of ALS $(381 \pm 52$ vs. $510 \pm 15 ; P<0.002)$ and IGFBP-3 $(112 \pm 14$ vs. $230 \pm 17 ; P<0.0001)$ and significantly higher levels of IGFBP-2 $(12.0 \pm 2.1$ vs. $5.7 \pm 0.6 ; P<0.005)$ than patients in whom surgery was unsuccessful. After successful surgery, 10 controlled patients showed normal concentrations of ALS, thus confirming good control of the disease, whereas one patient presented with an IGFBP-3 concentration slightly above the 97th percentile of the control group (Fig. 2 and Table 2). In 4 controlled patients, reevaluation after varying lengths of time (control 2) showed IGF-I, IGFBP-3, and ALS concentrations in the normal range.

Twenty-six of the 27 patients in whom surgery was unsuccessful had elevated IGF-I concentrations, whereas $2(7 \%)$ and $8(30 \%)$ showed normal ALS and IGFBP-3, respectively (Fig. 2 and Table 2). Reevaluation of the only patient who had presented with IGF-I, IGFBP-3, and ALS concentrations at the upper end of the normal range at the first control revealed increased concentrations of IGF-I and ALS, but not IGFBP-3. The other patient with ALS below the normal range, but IGF-I above the 97th percentile of the control group, showed 
TABLE 2. Sensitivity, specificity, and predictive values of IGF-I, IGFBP-3, and ALS in 37 acromegalic patients reevaluated 1-6 months after surgery

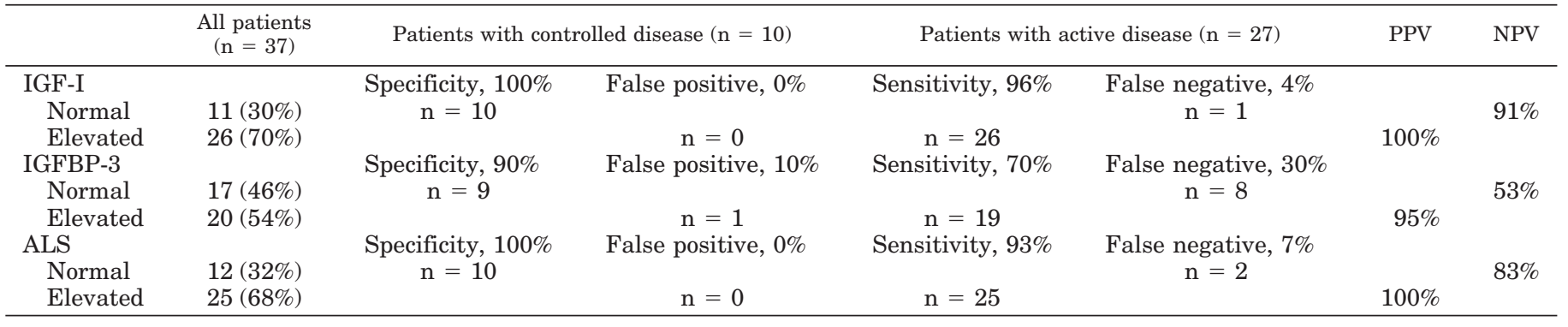

Data are given as the percentage of outcome of treatment. PPV and NPV, Positive and negative predictive values, respectively.

\section{BEFORE SURGERY}
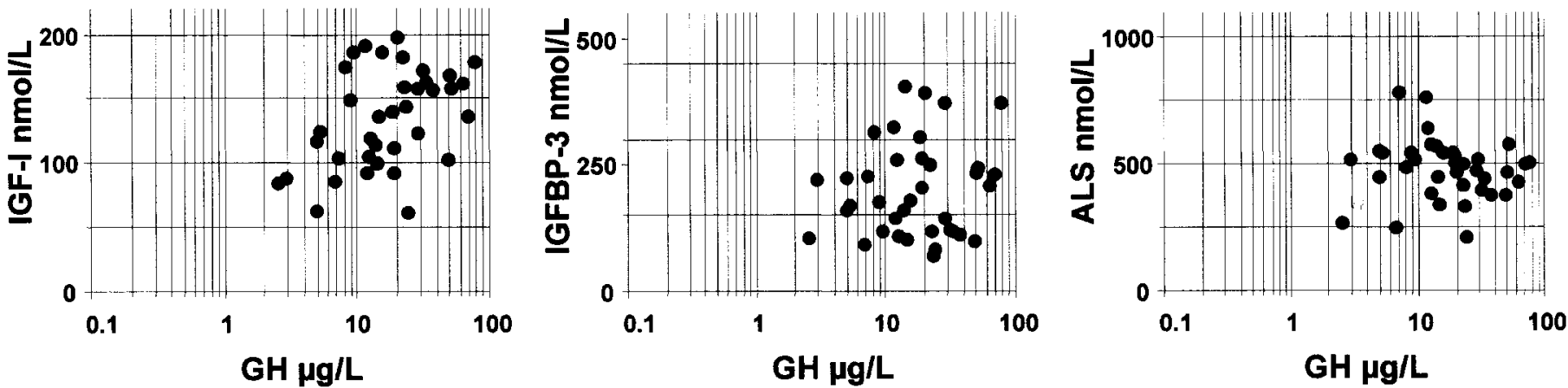

AFTER SURGERY

O controlled disease

active disease
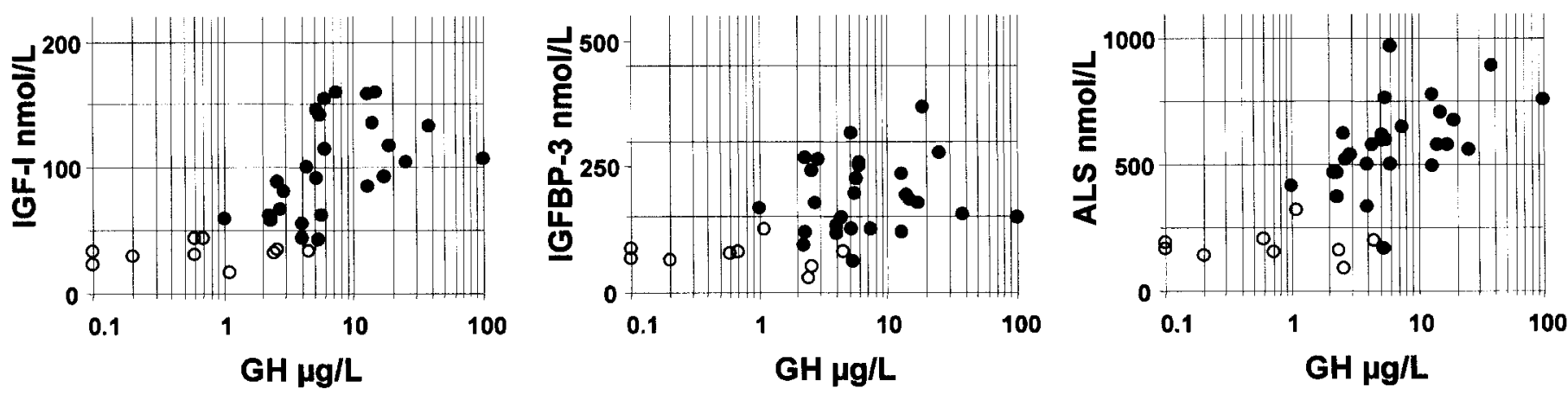

FIG. 4. Correlations between IGF-I, ALS, and IGFBP-3 concentrations and mean GH concentration in acromegalic patients before and after transsphenoidal surgery.

a nadir GH less than $1 \mu \mathrm{g} / \mathrm{L}$ after an OGTT and normal IGFBP-3. Reevaluation of this patient showed normalization of all parameters, including IGF-I concentration. Therefore, in 20 of 21 patients who were reevaluated (control 2), elevated concentrations of IGF-I and ALS persisted, ranging from 60-120 and from 382-976 nmol/L, respectively, whereas IGFBP-3 levels were normal in 5 patients.

IGFBP-1 levels increased slightly, although not significantly, in both controlled and uncontrolled patients (Fig. 1).

IGFBP-2 levels and IGFBP-2/IGF-I ratio increased significantly only in the group of controlled patients (Table 1 and Fig. 1). All patients with controlled disease showed a sig- nificantly increased $(P<0.001)$ IGFBP-2/IGF-I ratio in both postsurgical follow-up examinations, whereas 23 of 26 patients with active disease showed a ratio lower than the third percentile of that of the normal population.

In postsurgery samples (first control) a statistically significant $(P<0.001)$ correlation was found between mean $\mathrm{GH}$ values (Fig. 4) as well as minimum GH after OGTT and ALS $(\mathrm{r}=0.72$ and 0.83 , respectively), IGF-I $(\mathrm{r}=0.69$ and 0.77$)$, IGFBP-3 ( $\mathrm{r}=0.46$ and 0.67), and IGFBP-2 $(\mathrm{r}=-0.36$ and -0.63). Similarly, IGF-I, IGFBP-3, and ALS were positively correlated among themselves and negatively correlated with IGFBP-2 $(P<0.001)$. 


\section{Discussion}

As the subunits of the $150 \mathrm{~K}$ complex are GH dependent and stable in circulation, they constitute a sensitive and specific index of integrated 24-h GH secretion, and their use can avoid causing the patient the stress of multiple sampling for evaluation of the GH secretory state. Several studies have established the high specificity and sensitivity of the total IGF-I concentration in the diagnosis of acromegaly $(1,2,30)$, whereas data on the reliability of IGFBP-3 are conflicting, and those on ALS are scant, even though their measurement is less difficult, as it does not require previous extraction. Unfortunately, some well known limitations of IGF-I, resulting from its age dependency and its modification in some clinical conditions (malnutrition, diabetes, hypothyroidism, and chronic renal failure) are more or less shared by the other subunits of the $150 \mathrm{~K}$ complex. All of these limitations, however, can easily be overcome by means of specific testing for individual diseases and by defining normal ranges with reference to a large number of age- and sex-matched control subjects. In this respect it is noteworthy that ALS levels in 20to 65-yr-old normal subjects appear to be less age dependent than IGF-I and IGFBP-3. This finding is in agreement with the data reported by Juul et al. (31) in adult women, although we did not find a significant decrease in ALS levels in adult men. Our data in normal subjects confirm that the molar concentration of ALS in the circulation exceeds that of IGFBP-3 by about two thirds (23), and that its concentration is positively correlated with IGF-I and IGFBP-3 concentrations (25).

The present study, carried out by comparing a large number of acromegalic patients with 100 healthy adults, shows that active acromegaly is characterized by elevated ALS concentrations. Previous data are concordant with our finding that ALS levels in acromegalic patients are about twice those present in normal subjects. Indeed, using an antibody raised against serum-derived glycosylated ALS, Baxter (24) first showed that ALS levels were elevated in acromegalic patients and, using the same RIA, Hoffman et al. (15) reported mean ALS levels 2.5-fold above the normal mean and 91\% of patients with values above the normal range. More recently, using the same two-site sandwich enzyme-linked immunosorbent assay as we used, Khosravi et al. (25) found mean ALS levels in 20 acromegalics about 1.6-fold above the mean of control value, and $80 \%$ of patients above the normal range. In the 45 acromegalic patients evaluated in this study, pathological ALS levels were found in $89 \%$ of patients before treatment. In the remaining 5 patients, we could not find any known cause shared by all to justify ALS levels within the normal range. However, we cannot exclude that the complex regulatory mechanisms modulating the circulating levels of the 150K complex (16-22) might differently affect the synthesis of each subunit, at least in some patients. Nevertheless, in this subset of patients GH levels and IGF-I concentrations tended to be lower, and IGFBP-2 levels higher, than in patients with pathological ALS levels. The finding that all of them also showed IGFBP-3 below the normal range confirms the close association of these two components of the $150 \mathrm{~K}$ complex. Indeed, the findings that multiple linear regression analysis indicates that ALS is the factor most closely associated with IGFBP-3 concentration $(P<0.005)$ and that ALS/IGFBP-3 molar ratio found in acromegalic patients does not differ significantly from that seen in normal subjects are in agreement with the hypothesis that much of the apparent GH dependency of serum IGFBP-3 might be secondary to its stabilization by ALS (32). Considering the ALS/IGFBP-3 molar ratio, almost $55 \%$ of ALS should circulate in a free form in acromegaly. In agreement with data reported by Juul et al. (33), not only the IGF-I/IGFBP-3 molar ratio but also the IGF-I/IGFBPs $(-1+-2+-3)$ ratio, which can be regarded as free, biologically active, IGF-I, was greatly increased in patients with active acromegaly. This increase was more closely related to the increased IGF-I synthesis and the elevated IGF-I/IGFBP-3 molar ratio than to the reduction of the IGFBPs negatively regulated by GH. Indeed, IGFBP-2 was unchanged, and IGFBP-1 was, on the average, only half that seen in control subjects.

In the acromegalic patients before treatment, the levels of total IGF-I and IGFBP-3 were increased in 100\% and 73\% of patients, respectively. The finding regarding the poor reliability of IGFBP-3 in the diagnosis of acromegaly is in agreement with several previous reports showing that from $26-45 \%$ of patients have IGFBP-3 levels overlapping with those of healthy controls $(11,12,34,35)$, although in one study (36) elevated levels of IGFBP-3 were found in all 18 untreated acromegalic patients.

In postsurgical reassessment of the disease, it is noteworthy that ALS shows a positive predictive value equal to that of IGF-I (100\%) and a negative predictive value slightly lower $(83 \%)$ than that of IGF-I (91\%), whereas IGFBP-3 again shows the lowest sensitivity and specificity.

Although GH exerts an inhibitory effect on the synthesis of IGFBP-1 as well as IGFBP-2 $(29,38)$, IGFBP-1 is not usually considered, because, unlike IGFBP-3 and IGFBP-2, it shows marked diurnal variations due to changes in metabolic status (38). Moreover, owing to its short half-life and lower circulating levels, IGFBP-1 does not usually play an important role in stabilizing circulating IGFs (39). The observation that IGFBP-1 levels were lower in acromegaly than in normal subjects is in agreement with previous reports $(33,40,41)$ and with the known inhibition of this parameter by insulin (38). After surgery, IGFBP-1 levels increased, reaching statistical significance only in the group of controlled patients. Jørgensen et al. (41) found serum IGFBP-1 levels to be slightly supernormal after adenomectomy; this finding was not confirmed by our data, according to which IGFBP-1 only normalized.

Although IGFBP-2 levels significantly lower than those in healthy subjects have been reported in acromegalics $(33,41$, 42), our data, in agreement with those reported by Clemmons et al. (29), showed IGFBP-2 concentrations not significantly different from those found in a large group of normal subjects. With regard to the IGFBP-2/IGF-I molar ratio, $94 \%$ of patients showed a ratio below the third percentile of healthy subjects, and this ratio normalized in controlled patients. After surgery, IGFBP-2 greatly increased in the group of controlled patients, without any significant change in not controlled patients, thus suggesting that elevated $\mathrm{GH}$ and IGF-I indeed have an effect on IGFBP-2.

In postoperative samples, $\mathrm{GH}$ secretion (mean values as well as nadir GH after OGTT) correlated mostly with ALS and IGF-I and to a lesser extent with IGFBP-3. Our previous finding of a log-linear correlation between GH and IGF-I (2) is further corroborated by this study and extended to the ALS subunit and to IGFBP-3. 
In conclusion, our data show that active acromegaly is characterized by elevated ALS concentrations as well as elevated total and free IGF-I levels. Mean levels of IGFBP-3 were also higher, whereas levels of IGFBP-1 were lower than those in normal subjects. However, one quarter of the patients had IGFBP-3 levels in the normal range, and most of these showed normal IGFBP-1 and IGFBP-2 concentrations. These observations are equally true when considering newly diagnosed patients and patients with active disease after pituitary surgery. Therefore, in the diagnosis of acromegaly, the measurement of total IGF-I appears to be the most sensitive parameter among the subunits of the $150 \mathrm{~K}$ complex, and IGFBP-3 the least sensitive. For what concerns ALS, this subunit is seen to be quite sensitive and appears to be a useful parameter in reassessment after surgical treatment.

\section{References}

1. Clemmons DR, VanWyk JJ, Ridgway EC, Kliman B, Kjellberg RN, Underwood LE. 1979 Evalation of acromegaly by radioimmunoassay of somtomedin-C. N Engl J Med. 301:1138-1142.

2. Barreca A, Ciccarelli E, Minuto F, Bruzzi P, Giordano G, Camanni F. 1989 Insulin-like growth factor I and daily growth hormone profile in the assessment of active acromegaly. Acta Endocrinol (Copenh). 120:629-635.

3. Furlanetto RW. 1980 The somatomedin C binding protein: evidence for a heterologous subunit structure. J Clin Endocrinol Metab. 51:12-19.

4. Baxter RC. 1988 Characterization of the acid-labile subunit of the growth hormone-dependent insulin-like growth factor binding protein complex. J Clin Endocrinol Metab. 67:265-272.

5. Baxter RC, Martin JL, Beniac VA. 1989 High molecular weight insulin-like growth factor binding protein complex. Purification and properties of the acid-labile subunit from human serum. J Biol Chem. 264:11843-11848.

6. Barreca A, Ponzani P, Arvigo M, Giordano G, Minuto F. 1995 Effect of the acid-labile subunit on the binding of insulin-like growth factor (IGF)-binding protein-3 to [125I]IGF-I. J Clin Endocrinol Metab. 80:1318-1324.

7. Zapf J, Schmid CH, Froesch ER. 1984 Biological and immunological properties of insulin-like growth factors (IGF) I and II. Clin Endocrinol Metab. 13:3-30.

8. Daughaday WH, Kapadia M, Mariz IK. 1986 Serum somatomedin binding proteins: physiologic significance and interference in radioligand assay. J Lab Clin Med 109:335-363.

9. Mohan S, Baylink D. 1995 Development of a simple valid method for the complete removal of insulin-like growth factor (IGF)-binding proteins from IGFs in human serum and other biological fluids: comparison with acid-ethanol treatment and $\mathrm{C}_{18}$ Sep-Pak separation. J Clin Endocrinolo Metab. 80:637-647.

10. Rosenfeld RG, Gargosky SH. 1996 Assays of insulin-like growth factors and their binding proteins:practicalities and pitfalls. J Pediatr. 128:S52-S57.

11. De Herder WW, Van der Lely AJ, Janssen JAMJL, Uitterlinden P, Hofland LJ, Lamberts SWJ. 1995 IGFBP-3 is a poor parameter for assessment of clinical activity in acromegaly. Clin Endocrinol (Oxf). 43:501-505

12. Thissen JP, Ketelslegers JM, Maiter D. 1996 Use of insulin-like growth factor-I (IGF-I) and IGF-binding protein-3 in the diagnosis of acromegaly and growth hormone deficiency in adults. Growth Regul. 6:222-229.

13. de Boer H, Blok GJ, Popp-Snijders C, Stuurman L, Baxter RC, van der Veen E. 1996 Monitoring of growth hormone replacement therapy in adults, based on measurement of serum markers. J Clin Endocrinol Metab. 81:1371-1377.

14. Thoren M, Hilding A, Baxter RC, Degerblad M, Wivall-Helleryd IL, Hall K. 1997 Serum insulin-like growth factor I (IGF-I), IGF-binding protein-1 and -3, and the acid-labile subunit as serum markers of body composition during growth hormone $(\mathrm{GH})$ therapy in adults with GH deficiency. J Clin Endocrinol Metab. 82:223-228.

15. Hoffman DM, Baxter RC, O'Sullivan AJ, Crampton L, Ho K. 1997 Serum acid-labile subunit in adult growth hormone deficiency and acromegaly [Abstract]. Endocrinol Metab. 4(Suppl A):23.

16. Chin E, Zhou J, Dai J, Baxter RC, Bondy CA. 1994 Cellular localization and regulation of gene expression for components of the insulin-like growth factor ternary binding protein complex. Endocrinology. 134:2498-2504.

17. Dai J, Baxter RC. 1994 Regulation in vivo of the acid-labile subunit of the rat serum insulin-like growth factor-binding protein complex. Endocrinology. 135:2335-2341.

18. Ooi GT, Cohen FJ, Tseng LYH, Rechler MM, Boisclir YR. 1997 Growth hormone stimulates transcription of the gene encoding the acid-labile subunit (ALS) of the circulating insulin-like growth factor-binding protein complex and ALS promoter activity in rat liver. Mol Endocrinol. 11:997-1007.

19. Bereket A, Wilson TA, Blethen SL, et al. 1996 Regulation of the acid-labile subunit of the insulin-like growth factor ternary complex in patients with insulin-dependent diabetes mellitus and severe burns. Clin Endocrinol (Oxf) 44:525-532.

20. Barreca A, Ketelslegers JM, Arvigo M, Minuto F, Thissen JP. 1998 Decreased acid-labile subunit (ALS) levels by endotoxin in vivo and by interleukin- $1 \beta$ in vitro. Growth Horm IGF Res. 8:217-223.

21. Baxter RC, Hawker FH, To C, Stewart PM, Holman SR. 1997 Thirty-day monitoring of insulin-like growth factors and their binding proteins in intensive care unit patients. Growth Reg. 7:1-11.

22. Barreca A, Voci A, Lee PDK, et al. 1997 Effect of the somatostatin analog, octreotide, and of other hormones on the release of the acid-labile subunit (ALS) of the $150 \mathrm{kDa}$ complex by rat hepatocytes in primary culture. Eur J Endocrinol. 137:193-199.

23. Baxter RC. 1997 Editorial: the binding protein's binding protein: clinical ap plications of acid-labile subunit (ALS) measurement. J Clin Endocrinol Metab. 82:3941-3943.

24. Baxter RC. 1990 Circulating levels and molecular distribution of the acid-labile $(\alpha)$ subunit of the high molecular weight insulin-like growth factor-binding protein complex. J Clin Endocrinol Metab. 70:1347-1353.

25. Khosravi MJ Diamandi A, Mistry J, Krishna RG, Khare A. 1997 Acid-labile subunit of human insulin-like growth factor binding protein complex: measurement, molecular and clinical evaluation. J Clin Endocrinol Metab. 82:3944-3951

26. Giustina A, Barkan A, Casanueva FF, et al. 2000 Criteria for cure of acromegly: a consensus statement. J Clin Endocrinol Metab. 85:526-529.

27. Arosio M, Giovanelli MA, Riva E, Nava C, Ambrosi B, Faglia G. 1983 Clinical use of pre- and postsurgical evaluation of abnormal GH responses in acromegaly. J Neurosurg. 59:402-408

28. Daughaday WH, Mariz IK, Blethen SL. 1980 Inhibition of access of bound somatomedin to membrane receptor and immunoglobin sites: a comparison of radioreceptor and radioimmunoassay of somatomedin in native and acidethanol extracted serum. J Clin Endocrinol Metab. 51:781-788

29. Clemmons DR, Snyder DK, Busby Jr WH. 1991 Variables controlling the secretion of insulin-like growth factor binding protein-2 in normal human subjects. J Clin Endocrinol Metab. 73:727-733.

30. Lamberts SW, Uitterlinden P, Verleun T. 1987 Relationship between growth hormone and somatomedin-C levels in untreated acromegaly, after surgery and radiotherapy and during medical therapy with sandostatin (SMS 201-995). Eur J Clin Invest. 17:354-359.

31. Juul A, Møller S, Mosfeldt-Laursen E, et al. 1998 The acid-labile subunit of human ternary insulin-like growth factor binding protein complex in serum: hepatosplanchnic release, diurnal variation, circulating concentrations in healthy subjects, and diagnostic use in patients with growth hormone deficiency. J Clin Endocrinol Metab. 83:4408-4415.

32. Olivecrona H, Hilding A, Ekström C, et al. 1999 Acute and short-term effects of growth hormone on insulin-like growth factors and their binding proteins: serum levels and hepatic messenger ribonucleic acid responses in humans. J Clin Endocrinol Metab. 84:553-560.

33. Juul A, Main K, Blum WF, Lindholm J, Ranke MB, ND Skakkebaek NE. 1994 The ratio between serum levels of insulin-like growth factor (IGF)-I and the IGF binding proteins (IGFBP-1, 2 and 3) decreases with age in healthy adults and increases in acromegalic patients. Clin Endocrinol (Oxf). 41:85-93.

34. Paramo C, Andrade MAO, Fluiters E, Luna R, de la Fuente J, Garcìa-Mayor RV. 1997 Comparative study of insulin-like growth factor-I (IGF-I) and IGFbinding protein-3 (IGFBP-3) level and IGF-I/IGFBP-3 ratio measurements and their relationship with an index of clinical activity in the management of patients with acromegaly. Metabolism. 46:494-498

35. Stoffel-Wagner B, Springer W, Bidlingmaier F, Klingmüller D. 1997 A comparison of different methods for diagnosing acromegaly. Clin Endocrinol (Oxf). 46:531-537.

36. Grinspoon S, Clemmons D, Swearingen B, Klibanski A. 1995 Serum insulinlike growth factor-binding protein-3 levels in the diagnosis of acromegaly. J Clin Endocrinol Metab. 80:927-932.

37. Deleted in proof.

38. Lee PD, Giudice LC, Conover CA, Powell DR. 1997 Insulin-like growth factor binding protein-1: recent findings and new directions. Proc Soc Exp Biol Med. 216:319-357.

39. Zapf J. 1995 Physiological role of the insulin-like growth factor binding proteins. Eur J Endocrinol. 132:645-654

40. Holly JMP, Cotterill AM, Jemmott RC, et al. 1991 Inter-relations between growth hormone, insulin, insulin-like growth factor-I (IGF-I), IGF-binding protein-1 (IGFBP-1) and sex hormone-binding globulin in acromegaly. Clin Endocrinol (Oxf). 34:275-280.

41. Jørgensen JOL, Møller N, Møller J, Weeke J, Blum WF. 1994 Insulin-like growth factors (IGF)-I and -II and IGF binding protein-1, -2 , and -3 in patient with acromegaly before and after adenomectomy. Metabolism. 43:579-583.

42. Blum WF, Horn N, Kratzsch J, et al. 1993 Clinical studies of IGFBP-2 by radioimmunoassay. Growth Regul. 3:100-104. 